\title{
Involving "authentic" cancer patients, their caregivers, and multidisciplinary professionals in a quality improvement trajectory in a hospital cancer pathway: a study protocol
}

This article was published in the following Dove Press journal: Journal of Multidisciplinary Healthcare

Inge Melchior ${ }^{1,2}$

Albine Moser ${ }^{2,3}$

Marja Y Veenstra ${ }^{4}$

Kon-Siong Jie ${ }^{1,2}$

'Department of Internal Medicine, Zuyderland Medical Centre, Sittard, The Netherlands; ${ }^{2}$ Research Centre Autonomy and Participation of Chronically III people, Zuyd University of Applied Sciences, Heerlen, The Netherlands; ${ }^{3}$ Department of Family Medicine, CAPHRI, Maastricht University, Maastricht, The Netherlands; ${ }^{4}$ Burgerkracht Limburg [Citizen Power in Limburg], Sittard, The Netherlands
Correspondence: Kon-Siong Jie Department of Internal Medicine, Zuyderland Medical Centre, Postbus 5500, 6130 MB, Sittard, The Netherlands Email a.jie@zuyderland.nl
Introduction: The implementation of oncology care pathways that standardize organizational procedures has improved cancer care in recent years. However, the involvement of "authentic" patients and caregivers in quality improvement of these predetermined pathways is in its infancy, especially the scholarly reflection on this process. We, therefore, aim to explore the multidisciplinary challenges both in practice, when cancer patients, their caregivers, and a multidisciplinary team of professionals work together on quality improvement, as well as in our research team, in which a social scientist, health care professionals, health care researchers, and experience experts design a research project together.

Methods and design: Experience-based co-design will be used to involve cancer patients and their caregivers in a qualitative research design. In-depth open discovery interviews with 12 colorectal cancer patients, 12 breast cancer patients, and seven patients with cancer-associated thrombosis and their caregivers, and focus group discussions with professionals from various disciplines will be conducted. During the subsequent prioritization events and various co-design quality improvement meetings, observational field notes will be made on the multidisciplinary challenges these participants face in the process of co-design, and evaluation interviews will be done afterwards. Similar data will be collected during the monthly meetings of our multidisciplinary research team. The data will be analyzed according to the constant comparative method. Discussion: This study may facilitate quality improvement programs in oncologic care pathways, by increasing our real-world knowledge about the challenges of involving "experience experts" together with a team of multidisciplinary professionals in the implementation process of quality improvement. Such co-creation might be challenging due to the traditional paternalistic relationship, actual disease-/treatment-related constraints, and a lack of shared language and culture between patients, caregivers, and professionals and between professionals from various disciplines. These challenges have to be met in order to establish equality, respect, team spirit, and eventual meaningful participation.

Keywords: cancer care pathways, experience-based co-design, authentic cancer patients, patient involvement, caregivers' involvement, qualitative research

\section{Plain language summary}

It is generally the health care professional who designs the pathway in which cancer patients are treated in the hospital. Increasingly, professionals and scholars suggest that the quality of the care can only be improved if the actual users - the patients and their caregivers - become co-designers. The few researchers who have set up such projects, however, do not reflect in 
the scientific literature on the challenges they encounter in practice, which means that other scholars cannot learn from previous experiences. In this study protocol we would, therefore, like to tell what we aim to do with our study and which challenges we foresee.

We first want to do interviews with cancer patients and their caregivers, to gather their experiences in a large teaching hospital (Zuyderland MC, The Netherlands). Then we will interview the health care professionals, who are involved in their care, in group interviews. After that, we will bring the three groups together in one room during various meetings, and let them discuss which points they find most important to improve and how they want to solve them. As they are not used to work together, we expect several challenges, such as the various participants use different "languages", speak on different levels, and have different needs, such as the length and time of a meeting. We hope to build a team in which the unique background of every member will be of use instead of a constraint.

\section{Introduction}

In 2017 , approximately 109,663 people in the Netherlands were diagnosed with cancer, of which most have received treatment through clinical care pathways. ${ }^{1}$ The implementation of these pathways has led to an improved health care system, as they are "intended to reduce unnecessary practice variation, improve coordination and continuity, and ultimately improve outcomes of clinical care". ${ }^{2}$ The length of hospital stay has decreased, as a result, the costs have reduced and fewer in-hospital complications have been observed. ${ }^{3}$ However, this standardization of organizational procedures and evidence-based care also has an obvious drawback. Care pathways have mainly been developed by health care specialists, and have been diseases-based. ${ }^{4}$ Patients receive care in a predetermined path where patient involvement has only just started. ${ }^{2}$

Most literature on patient involvement focuses at the individual level, which means patients participate in decisions regarding their own care, ${ }^{5-7}$ known as shared-decision making. ${ }^{5,6}$ Collective patient involvement, on the other hand, refers mostly to (semi)professional "experience experts", such as members of patient organizations or client boards, and to patients' and caregivers' advocates, who participate in a project to improve health services. We define patient involvement as "the involvement of patients and family members in various activities concerning health care quality" in clinical cancer care pathways. ${ }^{8}$

Several studies have pointed out the importance of involving patients in quality improvement. ${ }^{9}$ Patients can help to improve patient information, access to services, and care environments. ${ }^{10}$ Tsianakas et $\mathrm{al}^{11}$ and Fudge et al ${ }^{12}$ argue that user involvement facilitates patient empowerment: the involved patients feel more confident as they are being heard by the clinicians and other people in a similar situation. Once they feel empowered, the users are more likely to voice themselves and have an impact on quality improvement. Their involvement has been most active in the areas with the least input from clinicians and in the least technical areas. Assuming that patient involvement leads to a higher level of patient satisfaction, all parties involved will benefit when patients' wishes and needs are more congruent with the health care system. Patients will be more intrinsically motivated, will recover more quickly, will be more compliant, and will experience fewer side effects. These benefits will lead to a reduction of costs, more satisfied patients, and more effective treatments. ${ }^{13}$

Despite growing enthusiasm and support for patient involvement in practice, a scholarly reflection on the involvement of patients and caregivers across a range of quality improvement activities in health care studies remains sparse. ${ }^{12,14}$ Dewar et $\mathrm{al}^{15}$ described the benefits of using the method of emotional touchpoints in order to improve care in a hospital setting, but they do not describe the barriers and facilitators to involve patients. A few previous studies have reflected on the facilitators of involving patients in a meaningful way: include patients and their caregivers from start to finish of the project, establish cohesion and respect among the participants, clarify the patient's role, sense of ownership of the project within the health care institution because staff members who believe in the project are essential for its success (institutional commitment), manage expectations of the participants and about the outcomes of the project, prepare and train staff prior to patient interviews, observe, shadow, and make a walkabout in the institution. ${ }^{16-21}$ Its barriers are the lack of time and engagement of physicians, institutional constraints, unfamiliarity with the participatory project design, difficulty finding patients who are willing to participate, a lack of time and engagement on the side of patients and caregivers, and staff discomfort with hearing the patient's voice (they are used to care for not with the patient). ${ }^{16-21}$

What remains unknown is whether these challenges of patient involvement in quality improvement also hold true in the case of "authentic" and more vulnerable cancer patients and their caregivers. With "authentic" patients we mean lay patients and caregivers who have experienced cancer care, but have no prior experience as patient representatives. Compared to experience experts, authentic patients who tell their story for the very first time, often find themselves in a more emotional and vulnerable state of being, and lack professional 
experience with patient representation. In practice, moreover, if lay patients are being involved at the collective level, they are often involved by consulting them about the health care quality, mainly in the area of service development and planning, ${ }^{8,22-24}$ and less with regard to actual quality improvement programs on the level of partnership. ${ }^{2,9,25}$ A critical reflection on involving authentic patients as partners in quality improvement, therefore, is what we will focus on in this article.

According to studies on multidisciplinary teamwork, the more diverse the backgrounds, languages, and "cultures" of the team members are, the more challenges a team faces in working toward a common goal and a positive team climate. ${ }^{26}$ Multidisciplinarity does not only concern the various professional disciplines, but also acknowledges "experience expertise" as adding yet another "disciplinary" background. We could expect that it takes an effort to create successful relationships, shared goals, shared knowledge, and mutual respect in a team where various health care professionals work together with individuals as vulnerable as elderly lay cancer patients. ${ }^{26}$ The latter often have a small social network, depend more on their families, are more likely to encounter comorbidity or multimorbidity problems, and have grown up having a paternalistic relationship with their doctors. ${ }^{27}$ Although it will be challenging to engage this group of vulnerable patients, their involvement in a co-design process provides us a unique insight in the working of a multidisciplinary team. As long as these challenges stay academically unreflected and undocumented, the chances that a researcher or professional will actually decide to involve authentic patients in quality improvement are not very high. In one of the few studies in which elderly patients were involved to improve outpatient services, there was no explicit reflection upon the participation in the quality improvement process. ${ }^{28}$

In this study protocol we will describe our quality improvement project - the structural integration of the experiences of cancer patients and their caregivers - as a vehicle to identify the multidisciplinary context of involving authentic cancer patients and caregivers as partners with professionals from multiple backgrounds in improving the quality of oncologic care pathways and of experience experts as partners in the project team.

\section{Design, methods, and analysis Research design}

The design we have chosen concerns participatory action research, more specifically, we used an experience-based co-design (EBCD). It is a qualitative study, in which in-depth interviews and focus group discussions will be conducted and extensive field notes will be taken. EBCD enables authentic patients to participate on a collective level as partners in the quality improvement process. EBCD focuses on the subjective experience of patients, caregivers, and health care professionals to identify key moments that shape a person's experience. These moments are called touchpoints. Bate and Robert explain experience-based design as follows:

By identifying the main areas (or 'touch points') where people come into contact with the service and where their subjective experience is shaped, [...] one can begin to design human experiences rather than just systems or processes. ${ }^{25}$

These experiences will be prominent in the co-design process that follows. The results of collective patient involvement do not disappear after one's personal treatment period is over, but will become an institutionalized part of the organizational culture. ${ }^{9,29}$ Patients, caregivers, and health care professionals will thus become co-designers of the health care services provided and will thus work together as a multidisciplinary team.

In total, the research process consists of five phases, as can be seen in Figure 1. Because EBCD is a process where the results of the previous phase will be the input for the next phase, we describe the process and data collection together.

\section{Setting}

The study will take place at the Zuyderland Medical Centre in Heerlen/Sittard. This hospital is located in the southern part of The Netherlands. In this area, $21 \%$ of the population consists of people older than 65 years $(17.4 \%$ is the overall Dutch average), making it one of the most aging regions of the Netherlands. The region, with its mining history, has, moreover, a majority living in lower social economic strata. ${ }^{30}$ Zuyderland Medical Centre is one of the largest urban teaching hospitals of The Netherlands, and in 2004 it signed a manifest called "contract with society" as a promise to invest more in patient-centered health care. ${ }^{31}$ In the clinical care pathways, every patient has a case manager (the primary contact person), who in most cases is a clinical nurse specialist. A case manager organizes the care around individual patients, provides information, and is involved in the aftercare of the patient.

\section{The research participants}

The research participants are lay cancer patients, their caregivers, and health care professionals. We will select 12 patients treated in the colorectal pathway (project 1) and 12 in the breast cancer pathway (project 2). They are all aged 65 years and older. We will try to include the most vulnerable 


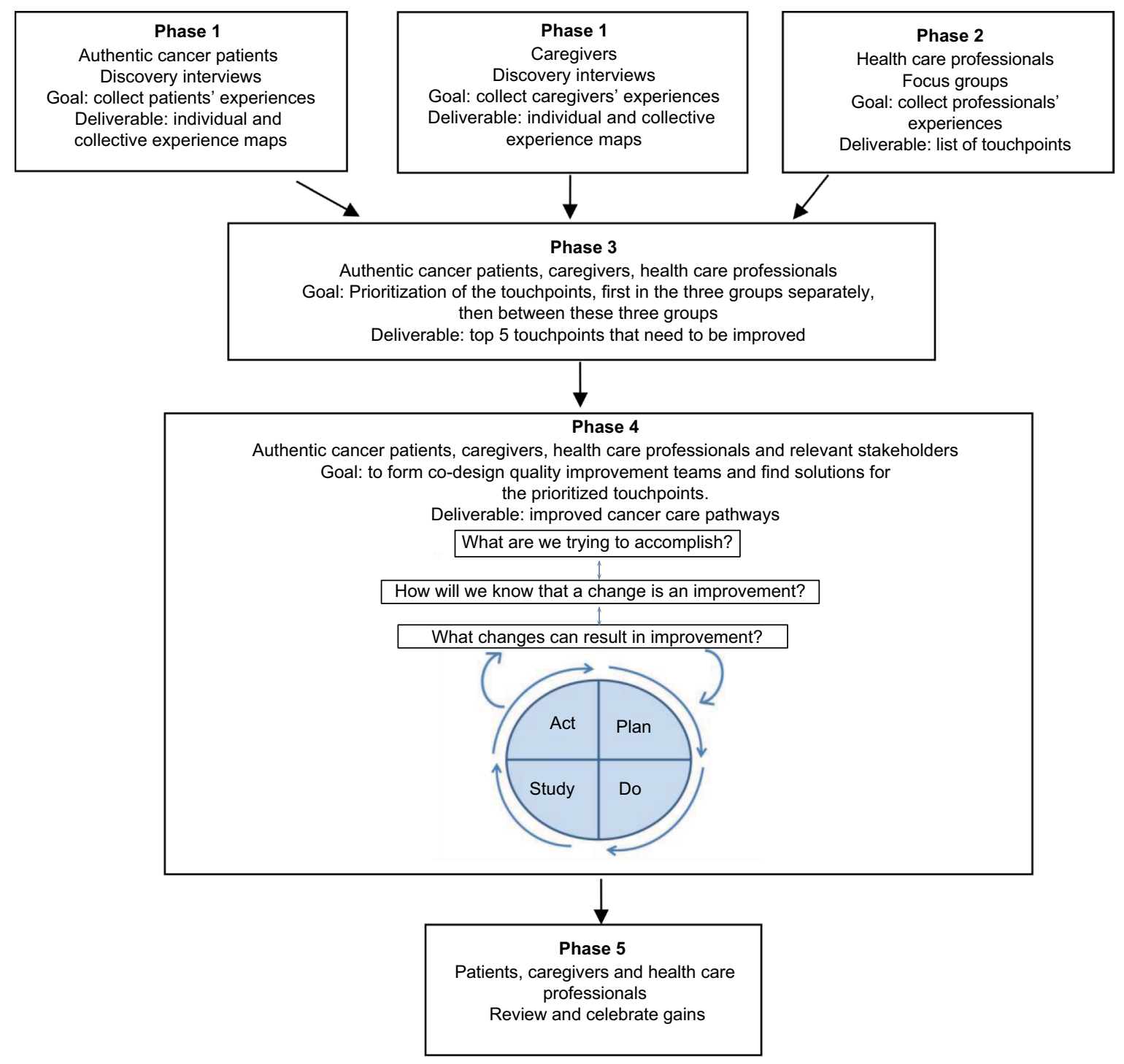

Figure I Experience-based co-design research process.

patients as well: the oldest, those with comorbidity problems, and those with hearing or seeing disabilities. We will try to select patients from various phases of the cancer care pathway. Those who are already in aftercare will only be asked if the treatment was less than 1 year ago. Being a patient in the palliative phase does not exclude one, as long as one is able to participate. To also include patients who fall outside the regular oncologic pathways, we will engage another seven oncologic patients who have cancer-associated thrombosis (CAT) (project 3). No further selection criteria will be made to include a wide variety of participants in our research.

Patients will be approached by the case manager during one of their face-to-face consultation meetings in the hospital. We aim at a random sampling of patients, not selected on their educational backgrounds or assertiveness. ${ }^{17,32}$ We explicitly chose to include lay patients and no patient representatives, as we want to stay as close to the actual experiences of people as possible (rather than their stories) and want to focus on exactly their "authentic" experiences with patient participation. We assume them to lack the (semi)professional "culture" that many patient representatives have already (partly) incorporated. Patients who participate will be asked to identify the spouse, relative, or friend most involved in their care. These 31 caregivers will also be invited to participate.

The health care professionals selected to participate will be those who are mentioned the most by the patients in the interviews, as they are apparently the most worth mentioning in terms of the patients' experience. We expect that they will have various disciplinary backgrounds, such as the oncologist, the pharmacist, the family doctor, physiotherapist, and 
the nurse. Our aim is to organize four focus group discussions per pathway with 6-8 professionals each.

\section{The research team}

The research team is composed of the project researcher (IM, an anthropologist with extensive experience in qualitative research methodology), two project leaders who will also be co-researchers (KSJ, a hematologist who is an associate professor in Patient-Centered Health Care, and AM, a senior nurse researcher experienced in patient involvement), and two patients' and caregivers' representatives (MYV and Esther Stoffers, staff members of Burgerkracht Limburg [Citizen Power in Limburg], a patient umbrella organization covering the whole southern region of the Netherlands). In project 3 we will also involve a cancer patient and a patient with thrombosis in our research team, in order to also learn from patient participation in research, plus a second researcher (Anouk van der Heijden, with a background in health sciences).

Furthermore, an advisory board consisting of representatives of the Integraal Kanker Centrum Nederland [Netherlands Comprehensive Cancer Organization] (IKNL), researchers with expertise in comorbidity and polypharmacy of elderly patients and primary care (Maastricht University), and a representative of the Netherlands Federation of Cancer Patients Organizations (NFK) are involved. Our research team consists of a wide range of disciplines and (professional and life) experiences, and offers a living-lab to study multidisciplinary teamwork.

\section{Data collection and procedure Phase I}

The project objective of phase 1 is to map the experiences of the cancer patients and their caregivers. By conducting in-depth interviews, interviewees will have the freedom to tell their own story. ${ }^{33}$ The anthropologist (IM, female, PhD) with extensive prior experience with ethnographic fieldwork and in-depth interviewing will start the interview with a very open question: could you please tell me how you have experienced your journey through the hospital? She will listen carefully and single out the touchpoints of the participants. These touchpoints are subsequently further explored by questions that are more probing: what did you experience at that moment, what made that experience good/bad, and how did that make you feel? The interviewer will also use an interview guide to ask the participants' experiences about the following topics: comorbidity, polypharmacy, treatment, communication, planning and information provision, and relationship with the general practitioner/home care. The choice for these topics is based on the requirement of the funding body. Special attention will be given to information provision in all phases of the patient's journey: diagnostics, treatment, and aftercare. The interviews will be video- and/or tape recorded. We will need these voice and film recordings in phase 3 , to confront the health care professionals with the "pure" stories of the patients and caregivers. These recordings are, thus, an essential part of the EBCD method.

A similar interview also takes place with each caregiver. The patient and his caregiver are interviewed separately if possible, to make sure that both voices are heard. However, if participants wish to do a couple interview, this wish is respected too, as it is of utmost importance that the interview setting feels natural and the participants feel at ease. That is also why the researcher will conduct the interviews in the participants' homes and will take her time to first get informally acquainted before starting the "official" interview. The researcher will also share her personal background and interests, in order to establish a relationship with the interviewee as equal and open as possible. The duration of the interview will be very flexible and depending on the interviewee.

The outcomes of phase 1 are individual "experience maps", which visualize the care pathway as experienced by each patient and caregiver, and the experiences they attach to each touchpoint in that pathway. Based on these individual experience maps, one collective experience map will be generated for each group of patients. That collective experience map will include all possible touchpoints for the specific group and relevant experiences. We will discuss and adjust it together with the patients and caregivers during phase 3 .

To answer the academic question central in this paper the researcher will make reflective observational notes on how patients and caregivers relate to their emotions, experiences, and stories when they narrate their journey in the hospital.

\section{Phase 2}

The project objective of phase 2 is to map the positive and negative experiences of the health care professionals in the cancer care pathways: what do they believe can be improved for these authentic cancer patients and their caregivers? This is accomplished by organizing focus group discussions to exchange and discuss experiences collectively. The questions will be directed at the same domains as in the interviews with the patients and caregivers: starting with an open question and then focusing on comorbidity, polypharmacy, information providence and planning, communication, treatment, and transmural care (with professionals outside of the hospital). Four focus group meetings are organized per clinical care pathway: one for the directly involved professionals in the hospital, one for those indirectly involved (eg, cardiologists, 
lung doctors), one for professionals involved from outside the hospital (eg, home care), and one mixed focus group discussion. Six to eight professionals are invited to each focus group meeting. These meetings will take place in the hospital to make participation easiest for the participants. The outcome of phase 2 is a list of touchpoints for the health care professionals, represented in categories, accompanied by citations of the professionals that illustrate each category. During these focus group discussions we will make notes on the interactions between professionals from different disciplines, in order to understand their differing and common values and goals.

\section{Phase 3}

The analyses of the data gathered in phases 1 and 2 will provide a list of key touchpoints for the authentic cancer patients, one for the caregivers, and one for the health care professionals of each of the three pathways (colorectal cancer, breast cancer, and CAT). The project objective of phase 3 is to prioritize these touchpoints as areas of quality improvement. This prioritization results after 12 (3 times 4) meeting events: the first three are with patients, caregivers, and professionals separately, and the fourth is a joint meeting event for colorectal cancer, breast cancer, and CAT each. This two-step approach guarantees full involvement of patients and caregivers, and equality in partnership among the three groups.

At the separate events, the researcher presents the main touchpoints by means of videotape fragments from the interviews from that particular group. If patients do not give permission to videotape the interview, other methods are used, for example, audio recordings or literal quotes. Subsequently, the experience maps are used to make a collective "emotional map". Participants walk along written touchpoints affixed to the wall and attach their emotions written on sticky notes to those moments that have touched them the most. The participants of each group then collectively decide which points they find essential to include in their list of priorities. In each of the three groups, six to eight people will participate. The outcome of these joint events is three priority lists with five issues essential to improve the quality of the cancer care pathways. The patients and caregivers will probably only realize during this first meeting that their stories are part of something bigger than themselves, namely that they are part of a "patients community" or "caregivers community".

Next, these priorities are collectively discussed by patients, caregivers, and health professionals, in a mixed joint event, preferably with the same participants who were involved in phases 1 and 2. At the beginning of the meeting the participants are shown film footage or audiotape fragments of the interviews in phase 1 , as a way to trigger their emotional response and create relationships between the participants. Subsequently, the three groups present their priority lists to each other. In dialog, they make a joint priority list with the agreement of all parties about the necessary and feasible improvements. These prioritization meetings will take place in the hospital.

During this phase, the researcher will make extensive notes on the facilitators and barriers to patient engagement. It is in this phase that authentic patients, their caregivers, and professionals will meet each other for the first time, discover each other as individuals, professionals, and each other's languages and cultures. It is also the start of their multidisciplinary teamwork.

\section{Phase 4}

The project objective of phase 4 is to actually design and implement quality improvement to clinical practice. Codesign working groups - in our study multidisciplinary quality improvement teams - are formed, in which (preferably the same) cancer patients, caregivers, and health care professionals are involved. Each team will work on one topic of improvement. These topics will be selected by urgency and feasibility. It will be important to manage expectations of what the team can actually accomplish during the duration of the study. We will focus on feasible and "visible" gains.

These co-design working groups will initiate, implement, and monitor the improvement by following the Plan, Do, Study, Act (PDSA) cycle. ${ }^{34}$ The co-design working groups are facilitated by quality improvement fellows working in the hospital. Important stakeholders such as employees in relevant management positions at the hospital will be involved in the co-design working groups as well to stay as close as possible to solutions that are promising for implementation and to prevent a clash with the solutions that the hospital is already working on. The outcome of this phase is the actual quality improvement in clinical practice. How this quality improvement will be measured is not yet fixed. It will depend on the decision made by the quality improvement team, whether improvements will focus on process, structure, or outcome of cancer care. We will work according to the PDSA cycle and perform a pre- and postevaluation.

This phase will provide the researcher with unique insights on the kind of cooperation and relationship, which is able to grow between lay patients, caregivers, and health care professionals in an EBCD project. The researcher will 
make extensive notes on the challenges that make multidisciplinary partnership in co-design difficult and its facilitating factors. At the end of phase 4 we will organize focus group discussions with the patients, caregivers, and professionals separately to conduct process evaluations. We will ask them to reflect on their experiences as part of the multidisciplinary cooperation in this project and lessons learned. Next to it, we hope to gain measurements of quality improvement activities.

\section{Phase 5}

The objective of the fifth and last phase is to make sure that the gains of the research project will not evaporate as soon as the project ends, to celebrate the gains and to evaluate the pitfalls. The latter will be done in an interactive workshop with all co-design groups. The outcome of this phase is a toolbox for the quality improvement department of the hospital to structurally involve authentic patients or experience experts in the improvement of the care they provide. The total project will take 3 years.

During the project we will also make extensive notes on the meetings of our own multidisciplinary research team. We will make self-reflective notes and discuss our barriers and facilitators together.

\section{Data analysis}

The interviews and focus group discussions are videotaped and transcribed verbatim. The extensive observational field notes written down during the entire co-design process and the interview transcripts will be analyzed using the analytical tool of constant comparative method based on the grounded theory approach. ${ }^{35}$ Data are read and re-read to get a general impression. Relevant text fragments are open coded, then compared and contrasted to formulate categories and subcategories according to the axial coding process. Software NVivo will be used to process the data. IM and Anouk van der Heijden will do the open coding processes. The coding process will be extensively discussed in our research team. We try to derive categories grounded in the data; however, we do keep the six topics required by the financing body in the back of our minds.

\section{Trustworthiness}

The four quality criteria for qualitative research - credibility, transferability, dependability, and conformability - are adhered to in order to safeguard the trustworthiness of the research results. ${ }^{36}$ First, member checks occur regularly to make sure that the participants agree with the analyses. Moreover, various kinds of data (cancer patients, caregivers, and health care professionals), methods (discovery interviews, focus group discussions, and field notes), and investigator triangulation (see research team) will be used to ensure credibility. Secondly, "thick descriptions" of the context of the particular patients, and the social settings in which the data were gathered, are used in order to inform other researchers about the extent to which the results are transferable to other cases and to show if they are dependent on a specific research context. Thirdly, the process of analysis is recurrently discussed within the research team to eliminate the danger that the findings depend too much on one interview or on the background of one researcher. Fourthly, the decision trail is reviewed to ensure that the study fulfills methodologic standards.

\section{Ethics approval and consent to participate}

The project is approved by the Ethics Commission of Zuyderland and Zuyd University of Applied Sciences. Participants will receive information before participation, get the opportunity to pose their questions, and sign an informed consent form. They will be able to withdraw from the project at any point. In the research presentations and reports, the anonymity of the patients and their caregivers will be guaranteed, having no reference to their names and to specific situations. The data will be handled confidentially and will only be available to the researchers of this research project.

\section{Discussion}

As with every research design, there are several points that deserve discussion. First, this research design heavily depends on the willingness to cooperate of the parties involved, on the "receptiveness of the local context". ${ }^{11}$ Health care professionals need to be open to new perspectives, especially the participatory approach of EBCD and able to appreciate the honesty and vulnerability of patients who share their personal stories. "Creating a safe environment for the patient so that she feels comfortable in exploring information and expressing opinions is probably the highest challenge for physicians who want to practice a shared approach". ${ }^{5}$ In addition, health care professionals themselves need to be prepared to open up as well, so that the patients and caregivers will feel equal. An important task is reserved here for the researcher, who will have to deal with the hospital hierarchies and power asymmetry between patients, caregivers, and health care professionals that are expected to be one of the barriers that such multidisciplinary team will encounter. We will use this quality 
improvement project to describe in detail which facilitators and barriers will be encountered in the creation of a fruitful cooperating relationship between authentic cancer patients, caregivers, and professionals.

Secondly, because the project depends on the participation of cancer patients who are either in treatment or have just finished treatment, the physical and emotional health of the patient will be a crucial component in this project. Although we ideally hope to involve patients and caregivers from beginning to end, we can expect that several of them, more than in average research, will drop out along the way. We hope to partly cover for this dropout by including more than one patient, by involving the patient advocates in our research team, and by including the experiences of the caregivers, as this ensures that the voice of the patient is being heard and represented as closely as possible. ${ }^{14}$ Besides that, the touchpoints that are selected from the interviews and prioritized in phase 3 and further are not individual topics, but collective touchpoints that recur in several interviews. This not only improves the quality of the research, but also makes it possible for new patients to step in for patients who are unable to continue after phase 1 . We will investigate what this shift of participants means for the teamwork and implementation process that follows and thereby what facilitators and barriers play a role.

Third, it should be acknowledged that it might not be without reason that elderly cancer patients, an age group for which most improvements could actually be gained, ${ }^{37}$ has remained largely underrepresented in research on patient involvement. These patients grew up in a time when a relationship with health care professionals was commonly paternalistic, so there may be a generational and thus cultural barrier to speaking up to their doctors. In addition, aging brings along a "loss of status as full persons", which might be accelerated by sickness and dependency. Elderly people who become patients might find it more difficult than younger patients to communicate their wishes and suggestions to the health care professional, simply because they themselves might not know how they perceive their future. ${ }^{38}$ It can be expected that these characteristics of elderly patients not only influence individual but also collective patient participation. They are not used to speaking with health care professionals as equals.

The question, however, is whether we will actually be able to involve the "harder to reach" patient groups remaining unheard so far. ${ }^{11}$ It might be very likely that the most vulnerable patients will decline our invitation for participation. We will, however, do our best to make the conditions for participation as facilitating as possible. We will work on trusting relationships and hospitality (for example provide soup and sandwiches during the joint meetings), give them vouchers to reward their time investment, and make sure their travel costs will be reimbursed and a taxi-bus will be offered to ease accessibility. We will give them control over participation and try to prevent overload: they will be asked after each phase whether they want to participate in the next phase. For example, they can decide to participate in the interview, but not continue with phase 3 . Once they continue to participate, we will make sure that the patients and caregivers are being supported by the two patient advocates in our research team (Burgerkracht Limburg). In cooperation with a facilitator from the hospital, we as researchers will assure equality between the various participants in the project. We will have preparatory meetings with the patients and caregivers before the co-design meetings to place them in a stronger position and make them feel valuable and welcome. However, the question how to deal with these patients' vulnerability is one of the questions we need to explore in this project.

Fourthly, in the relatively short timeframe of the project we might be able to study only part of the quality improvement cycle in depth. As we do not define the quality improvement topics and their complexity beforehand, the actions for improvement might take longer than the project. It is possible that we will not be able to study facilitators and barriers for the quality improvement cycles for all of the topics completely. This might also impede on observing the outcomes of the quality improvement cycles and studying facilitators and barriers in this special moment in the implementation of quality improvement.

A fifth point for discussion concerns the design of patient involvement. It can be questioned whether patient involvement and multidisciplinary teamwork is desirable and essential at every research stage. For instance, we intend to involve patients at every stage. However, there was no patient or caregiver involved in writing the research proposal. The reason is that we did not have patient researchers at our disposal. We did make use of patient advocates (staff members of Burgerkracht Limburg). Moreover, we choose to work with authentic patients in phase 3 and 4, which may cause problems in the implementation phase when patients need to be able to transcend their personal stories and think in more abstract terms, especially given their age and lack of experience in patient representation. To support them in this process, we will again involve patient advocates (staff members of Burgerkracht Limburg), a buddy of cancer patients, and in project 3 also the experience experts partici- 


\begin{tabular}{|c|c|c|c|c|c|c|c|c|c|c|c|}
\hline & \multicolumn{2}{|l|}{ 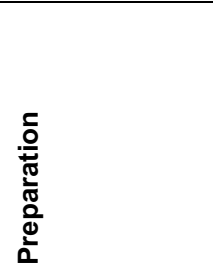 } & \multicolumn{4}{|l|}{$\begin{array}{l}\bar{\Phi} \\
\mathbb{d} \\
\bar{\pi} \\
\bar{\alpha}\end{array}$} & \multicolumn{3}{|l|}{ 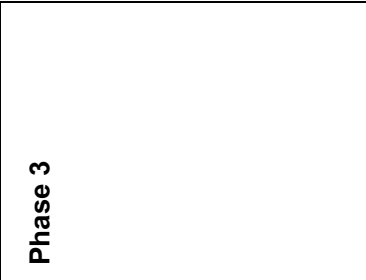 } & \multirow{2}{*}{ 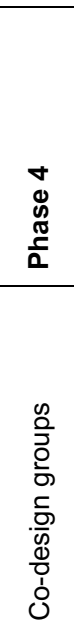 } & \multirow{2}{*}{ 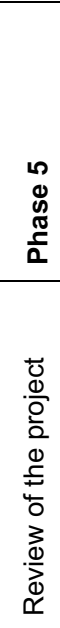 } \\
\hline & 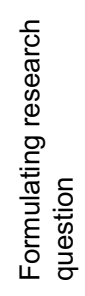 & 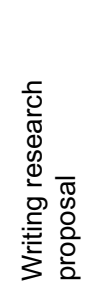 & 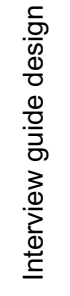 & 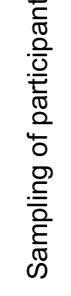 & 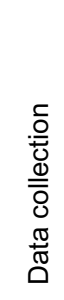 & $\begin{array}{l}\frac{\infty}{\infty} \\
\frac{0}{\pi} \\
\frac{\pi}{\pi} \\
\tilde{\pi} \\
\frac{\pi}{\pi} \\
0\end{array}$ & 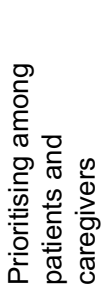 & 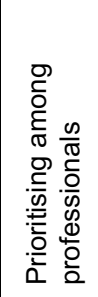 & 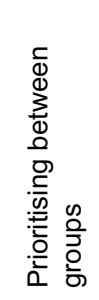 & & \\
\hline Patient control & & & & & & & $\mathrm{X}^{2,3}$ & & & & \\
\hline Partnership & & & & & & & & & $x^{2,3}$ & $x^{2,3}$ & $\mathrm{X}^{2,3}$ \\
\hline Advise & & $\mathrm{X}^{1}$ & $X^{1,5}$ & $X^{1,5}$ & $\mathrm{X}^{1,5}$ & $X^{1,5}$ & $X^{1,5}$ & $\mathrm{X}^{1,5}$ & $\mathrm{X}^{1,5}$ & $\mathrm{X}^{1,5}$ & $\mathrm{X}^{1,5}$ \\
\hline Consultation & $\mathrm{X}^{1}$ & & $\mathrm{X}^{4}$ & & & $x^{4}$ & & & & $x^{4}$ & $x^{4}$ \\
\hline Information & & & & & & & & $x^{2,3}$ & & & \\
\hline
\end{tabular}

Figure 2 Planned patient involvement (as co-designers).

Notes: I= staff members of the patient umbrella organisation Burgerkracht Limburg (Citizen Power in Limburg), participating as patient advocates; $2=$ authentic cancer patients; $3=$ family members of authentic cancer patients; $4=$ buddy of cancer patients; $5=$ (former) patients with cancer/thrombosis, participating as patient representatives in the research team. Figure based on the participation ladder and theoretical thinking of Arnstein. ${ }^{39}$

pating in our research team. At the end of the proposed study we will reflect upon the question: what is the advantage of working with these "authentic" patients and what are the challenges in involving them in multidisciplinary quality improvement? Figure 2 shows how we plan to involve the patients in each phase of the project. ${ }^{39}$

Finally, previous EBCD research has pointed out that what makes the project so valuable is the very experience of participating in the project. ${ }^{19}$ This raises the question whether the results can be successfully communicated to those professionals who did not participate in such multidisciplinary team. If not, implementation on the long run might not be successful, as the project might not feel as "owned" by the institution. A simple report might not be as convincing as having the direct experience of equal partnership with patients and caregivers. As we explicitly aim to transfer our insights to other cancer care pathways and health care settings, we will need to find ways to transmit the emotional experience of participation in the project and the bond that the multidisciplinary team members establish during the project to nonparticipants in order to be able to establish sound longterm implementation strategies.

\section{Acknowledgments}

This work was supported by The Dutch Cancer Society (KWF kankerbestrijding) grant number ATR2013-6458, Pfizer, and Leo Pharma.

\section{Author contributions}

All authors contributed toward data analysis, drafting and critically revising the paper, gave final approval of the version to be published, and agree to be accountable for all aspects of the work.

\section{Disclosure}

The authors report no conflicts of interest in this work.

\section{References}

1. Nederlandse Kankerregistratie, Integraal Kankercentrum Nederland, c2011-2018 [updated 2018 February 2]. Available from: https://www. cijfersoverkanker.nl. Accessed June 5, 2018. 
2. Faber MJ, Grande S, Wollersheim H, Hermens R, Elwyn G. Narrowing the gap between organisational demands and the quest for patient involvement: the case for coordinated care pathways. Int J Care Coord. 2014;17(1-2):72-78.

3. Rotter T, Kinsman L, James E, et al. Clinical pathways: effects on professional practice, patient outcomes, length of stay and hospital costs. Cochrane Database Syst Rev. 2010;3(3):CD006632.

4. Røsstad T, Garåsen H, Steinsbekk A, Sletvold O, Grimsmo A. Development of a patient-centred care pathway across healthcare providers: a qualitative study. BMC Health Serv Res. 2013;13:121.

5. Charles C, Gafni A, Whelan T. Decision-making in the physician-patient encounter: revisiting the shared treatment decision-making model. Soc Sci Med. 1999;49(5):651-661.

6. Friesen-Storms JH, Bours GJ, van der Weijden T, Beurskens AJ. Shared decision making in chronic care in the context of evidence based practice in nursing. Int J Nurs Stud. 2015;52(1):393-402.

7. Parsons E, Atkinson P. Lay constructions of genetic risks. Sociol Health Illn. 1992;14:437-455.

8. Crawford MJ, Rutter D, Manley C, et al. Systematic review of involving patients in the planning and development of health care. BMJ. 2002;325(7375):1263-1267.

9. Robert G, Cornwell J, Locock L, Purushotham A, Sturmey G, Gager M. Patients and staff as codesigners of healthcare services. BMJ. 2015;350:g7714.

10. Attree P, Morris S, Payne S, Vaughan S, Hinder S. Exploring the influence of service user involvement on health and social care services for cancer. Health Expect. 2010;14:48-58.

11. Tsianakas V, Robert G, Maben J, et al. Implementing patient-centred cancer care: using experience-based co-design to improve patient experience in breast and lung cancer services. Support Care Cancer. 2012;20(11):2639-2647.

12. Fudge N, Wolfe CD, Mckevitt C. Assessing the promise of user involvement in health service development: ethnographic study. $B M J$. 2008;336(7639):313-317.

13. Ahmed F, Burt J, Roland M. Measuring patient experience: concepts and methods. Patient. 2014;7(3):235-241.

14. Craig GM. Involving users in developing health services. BMJ. 2008;336(7639):286-287.

15. Dewar B, Mackay R, Smith S, Pullin S, Tocher R. Use of emotional touchpoints as a method of tapping into the experience of receiving compassionate care in a hospital setting. J Res Nurs. 2010;15(1):29-41.

16. Bak K, Macdougall L, Green E, et al. Hindsight is 20/20: lessons learned after implementing experience based design. PXJ. 2014;1(2):12-19.

17. Renedo A, Marston C, Spyridonidis D, Barlow J. Patient and public involvement in healthcare quality improvement: how organizations can help patients and professionals to collaborate. Public Manag Rev. 2015;17(1):17-34.

18. Armstrong N, Herbert G, Aveling EL, Dixon-Woods M, Martin G. Optimizing patient involvement in quality improvement. Health Expect. 2013;16(3):e36-47.

19. Donetto S, Tsianakas V, Robert G. Using experience-based co-design (EBCD) to improve the quality of healthcare: mapping where we are now and establishing future directions. London: King's College London; 2014.

20. Bossen C, Dindler C, Iversen OS, editors. Impediments to User Gains: Experiences from a Critical Participatory Design Project. Proceedings of the Participatory Design Conference. New York: ACM Press; 2012:31-40.
21. Bowen S, Mcseveny K, Lockley E, Wolstenholme D, Cobb M, Dearden A. How was it for you? Experiences of participatory design in the UK health service. CoDesign. 2013;9(4):230-246.

22. Mockford C, Staniszewska S, Griffiths F, Herron-Marx S. The impact of patient and public involvement on UK NHS health care: a systematic review. IJQHC. 2012;24:28-38.

23. Daykin NE, Evans D, Petsoulas C, Sayers A. Evaluating the impact of patient and public involvement initiatives on UK health services: a systematic review. Evid Policy. 2007;3:47-65.

24. Reed J, Cook G, Bolter V, Douglas B. Older people involved in policy and planning: factors which support engagement. J Aging Stud. 2008;22:273-281.

25. Bate P, Robert G. Bringing user experience to healthcare improvement: the concepts, methods and practices of experience-based design. Oxford/New York: Radcliffe Publishing; 2007.

26. Hartgerink JM, Cramm JM, Bakker TJ, van Eijsden AM, Mackenbach JP, Nieboer AP. The importance of multidisciplinary teamwork and team climate for relational coordination among teams delivering care to older patients. J Adv Nurs. 2014;70(4):791-799.

27. Balducci L, Yates J. General guidelines for the management of older patients with cancer. Oncology. 2000;14(11A):221-227.

28. Wolstenholme D, Cobb M, Bowen S, Wright P, Dearden A. Design led service improvement for older people. $A M J$. 2010;3(8):465-470.

29. Bate P, Robert G. Toward more user-centric OD: lessons from the field of experience-based design and a case study. J Appl Behav Sci. 2007;43:41-66.

30. Statistics Netherlands. CBS StatLine. Available from: https://opendata. cbs.nl/statline/\#/CBS/nl/. Accessed September 20, 2018.

31. Zuyderland MC; Atrium MC. Contract met de samenleving: het eeuwfeest (2004) als startpunt [Contract with society: the century festivities (2004) as a starting point]; 2004. Available from: https:// www.atriummc.nl/home/patienten-bezoekers/patientenservice/contractmet-de-samenleving/ contract-met-de-samenleving/. Accessed October 4, 2018. Dutch.

32. El Enany N, Currie G, Lockett A. A paradox in healthcare service development: professionalization of service users. Soc Sci Med. 2013;80:24-30.

33. Russell BH. Research Methods in Anthropology: Qualitative and Quantitative Approaches. Lanham, MD: AltaMira Press; 2006.

34. Taylor MJ, Mcnicholas C, Nicolay C, Darzi A, Bell D, Reed JE. Systematic review of the application of the plan-do-study-act method to improve quality in healthcare. BMJ Qual Saf. 2014;23(4): 290-298.

35. Strauss A, Corbin J. Basics of Qualitative Research: Grounded Theory Procedures and Techniques. Newbury Park, CA: Sage; 2008.

36. Lincoln YS, Guba EG. Naturalistic Inquiry. Newbury Park, CA: Sage Publications; 1985.

37. Cook G, Klein B. Involvement of older people in care, service and policy planning. J Clin Nurs. 2005;14(Suppl 1):43.

38. Latimer J. The dark at the bottom of the stairs: performance and participation of hospitalized older people. Med Anthropol $Q$. 1999;13(2):186-213.

39. Arnstein SR. A ladder of citizen participation. J Am Inst Plann. 1969; 35(4):216-224 


\section{Publish your work in this journal}

The Journal of Multidisciplinary Healthcare is an international, peerreviewed open-access journal that aims to represent and publish research in healthcare areas delivered by practitioners of different disciplines. This includes studies and reviews conducted by multidisciplinary teams as well as research which evaluates the results or conduct of such teams or health care processes in general. The journal covers a very wide range of areas and welcomes submissions from practitioners at all levels, from all over the world. The manuscript management system is completely online and includes a very quick and fair peer-review system. Visit http://www.dovepress.com/ testimonials.php to read real quotes from published authors.

Submit your manuscript here: https://www.dovepress.com/journal-of-multidisciplinary-healthcare-journal 